\title{
Constraints on the surface materials of Ryugu from spectral and water analysis of experimentally-heated CI carbonaceous chondrite
}

\author{
K. AMANO ${ }^{1 *}$, T. NAKAMURA ${ }^{1}$, F. USUI ${ }^{2}$, S. OKUMURA ${ }^{1}$, \\ AND M. E. ZOLENSKY ${ }^{3}$ \\ ${ }^{1}$ Department of Earth Science, Graduate School of Science, \\ Tohoku University, Sendai, Miyagi 980-8578, Japan. \\ (amakana@dc.tohoku.ac.jp) \\ ${ }^{2}$ Center for Planetary Science, Graduate School of Science, \\ Kobe University, Kobe, Hyogo 650-0047, Japan \\ ${ }^{3}$ Astromaterials Research and Exploration Science, NASA \\ Johnson Space Center, Houston, TX 77058, USA.
}

We performed heating experiments of the Orgueil CI chondrite in vacuum conditions at $\mathrm{fO}_{2}$ around $\mathrm{I} / \mathrm{W}$ to evaluate systematic changes in mineralogy, water contents, and visible to infrared spectral features. Our goal is to interpret the spectra of C-type asteroid Ryugu, the target of the Hayabusa2 mission, because Ryugu shows spectral features similar to partially dehydrated carbonaceous chondrites $[1,2]$. However heated carbonaceous chondrites can be easily contaminated by terrestrial water, which makes it difficult for laboratory data to be compared with spectra of asteroids directly. Therefore, our heated samples were analyzed without exposure to the air after experimental heating to minimize the effects of terrestrial water.

The depth of the 2.7- $\mu \mathrm{m}$ absorption band and the water content of the heated Orgueil form a positive correlation trend which follows the results of experimental heating of CM and Tagish Lake meteorites using our experimental procedures [3][4].

It is found that $500-{ }^{\circ} \mathrm{C}$ heated Orgueil sample shows the best spectral match with Ryugu in terms of the low reflectance $(\sim 2 \%)$ at visible wavelengths and a weak absorption $(\sim 10 \%)$ at around $2.72 \mu \mathrm{m}$ owing to survival of Mg-rich phyllosilicates. We suggest that Ryugu might have experienced heating and dehydration at a temperature around $500{ }^{\circ} \mathrm{C}$, after aqueous alteration, and presently contains a small quantity of hydrated minerals equivalent to $1-2 \mathrm{wt} . \%$ of water at the surface.

[1] Kitazato et al., (2019). Science 364, 272-275.

[2] Sugita et al., (2019). Science 364, eaaw0422.

[3] Mogi et al., (2017). The 80th Annual Meeting of the Meteoritical Society, New Mexico, Abstract \#6225.

[4] Amano et al., (2018). The 81st Annual Meeting of the Meteoritical Society, Moscow, Abstract \#6309. 